\title{
Rapid multiplex gene expression assays for monitoring metabolic resistance in the major malaria vector Anopheles gambiae
}

\author{
Konstantinos Mavridis ${ }^{1 *}$, Nadja Wipf ${ }^{2,3}$, Sandrine Medves ${ }^{4}$, Ignacio Erquiaga ${ }^{4}$, Pie Müller $^{2,3}$ and John Vontas ${ }^{1,5^{*}}$
}

\begin{abstract}
Background: Metabolic resistance of the major malaria vector Anopheles gambiae (s.l.) to insecticides is operationally significant, particularly in combination with target site resistance. However, detection of metabolic resistance is not trivial and relies on laborious bioassays, unspecific biochemical methods, or sophisticated and expensive molecular approaches using transcriptomics.
\end{abstract}

Methods: Rapid one-step multiplex TaqMan-probe based RT-qPCR assays were developed and optimised to measure the expression levels of genes associated with metabolic insecticide resistance in An. gambiae (s.l.). Primers and probes were designed to target the mRNA of cytochrome P450-dependent monooxygenases CYP6P3, CYP6M2, CYP9K1, CYP6P4 and CYP6Z1, and the glutathione-S-transferase GSTE2. The novel assays were validated versus gold standard methods with a range of phenotyped mosquito specimens. The assays were also tested directly on lysates of RNAlater ${ }^{\oplus}$-preserved mosquitoes without an RNA extraction step.

Results: The novel assays are efficient (reaction efficiencies $=95-109 \%$ ), sensitive (covering a $>10.0 \mathrm{Ct}$ range with $R^{2}$ values $>0.99$ ), specific (TaqMan chemistry), reproducible (\%CV $\left.=4.46-12.07 \%\right)$, as well as readily expandable to capture additional loci as they evolve or to cover additional species. The assays were successfully validated in terms of expression levels against standard two-step singleplex qPCR assays (overall \% difference $=-17.6 \%, 95 \% \mathrm{Cl}=-38.7-3.43 \%$ ) and microarrays, using laboratory strains and field-caught samples. The assays can also be applied directly on lysates of mosquito specimens, without RNA extraction or DNase treatment.

Conclusions: The novel multiplex assays for monitoring the levels of major detoxification genes and metabolic resistance in An. gambiae (s.l.) are simple to perform, robust and rapid. They may complement current diagnostic assays to provide evidence-based and operationally relevant information for insecticide resistance management.

Keywords: Multiplex TaqMan assays, Detoxification, P450s, GSTEs, Insecticide resistance, Metabolic resistance, Gene expression

\section{Background}

Insecticide based vector control interventions have reduced malaria incidence [1]. However, the increasing use of a limited number of insecticides, primarily pyrethroids, places an immense selection pressure on insect populations, which has not left disease vectors unaffected [2,3]. The resulting insecticide resistance (IR) in the major malaria vector Anopheles gambiae (s.l.) represents one of the greatest

\footnotetext{
*Correspondence: konstantinos_mavridis@imbb.forth.gr;

vontas@imbb.forth.gr

${ }^{1}$ Institute of Molecular Biology and Biotechnology, Foundation for Research and Technology-Hellas, 70013 Heraklion, Greece

Full list of author information is available at the end of the article
}

challenges in malaria control. In Anopheles mosquitoes, resistance is primarily conferred by mutations at the insecticide's target site that alter its sensitivity, and by the upregulation of enzymes that detoxify or sequester the insecticide [4]. Several cytochrome P450-dependent monooxygenases have been functionally associated with pyrethroid resistance. CYP6P3 [5] and CYP6M2 [6] are considered as the main pyrethroid metabolising enzymes in several $A n$. gambiae populations in West Africa. CYP6Z1 is associated with both pyrethroid and dichlordiphenyltrichlorethan (DDT) resistance [7]. CYP6P4 is associated with resistance to both alpha-cyano and non-alpha-cyano pyrethroids [8],

(C) The Author(s). 2019 Open Access This article is distributed under the terms of the Creative Commons Attribution 4.0 International License (http://creativecommons.org/licenses/by/4.0/), which permits unrestricted use, distribution, and 
and metabolises the juvenile hormone pyriproxyfen that is used as insect growth regulator by preventing larvae from developing into adult stages [9]. CYP9K1 was recently found to be overexpressed in deltamethrin-resistant Bioko populations and also metabolises pyrethroids [10]. The glutathione-S-transferase GSTE2 is associated with DDT [11] and pyrethroid [12] resistance.

Up-to-date data on resistance to insecticides are a prerequisite for the effective implementation of interventions. While a multitude of high throughput assays for the detection of individual target site resistance mutations have been developed and used to facilitate the implementation of vector control strategies $[13,14]$, there are very few such tools available for monitoring metabolic insecticide resistance in mosquito field populations [12]. In the absence of DNA markers associated with overexpression of detoxification genes for An. gambiae (s.l.), detection methods for metabolic resistance are restricted to either phenotypic bioassays with synergists or biochemical assays. Synergists are compounds that inhibit metabolic detoxification enzymes. By comparing the mortality of mosquitoes exposed to a synergist followed by the insecticide to the mortality of mosquitoes exposed to only the insecticide, a conclusion can be drawn as to whether and which enzyme families are involved in the observed resistance [15]. Two major drawbacks of synergist-insecticide bioassays are that they require large numbers of live mosquitoes and that they may only indicate which enzyme class (e.g. P450s, GSTs or esterases) is implicated and not specify which one of the enzymes is overexpressed. Biochemical measurement of resistance enzyme activity on microplates represents a simpler alternative to phenotypic response-to-exposure tests [16]. However, the biochemical assays' generic substrates are recognised by several members in an enzyme family and, therefore, are equally non-specific. Synergist-insecticide bioassays and biochemical assays are currently recommended follow-up techniques to determine the resistance mechanisms in resistant mosquito populations [17]. These methods have indeed been useful to associate elevated levels of mixed function oxidases with pyrethroid resistance in the field, for example in An. gambiae from Kenya [18] and in An. funestus from southern Africa [19] but they could not specify which P450s were overexpressed. In an attempt to have more specific assays available, chromo- and fluorogenic substrates with a higher preference for insecticide "metabolisers" have been identified or synthesised in some cases [20,21], with relatively restricted applicability because of the limited availability of substrates and/or complexity of biochemical reactions.

We believe that multiplex TaqMan assays are the future for resource-efficient and high-throughput monitoring of metabolic resistance in mosquito field populations. Here, we developed novel and rapid multiplex detoxification gene expression assays suitable for monitoring the specific upregulation of genes responsible for metabolic resistance in Anopheles mosquitoes. These qPCR assays were designed in the framework of the interdisciplinary research project DMC-MALVEC (dmc-malvec.eu) with the aim to develop an automated diagnostic platform (LabDisk) for malaria vectors [22], though they can also be applied independently in conventionally prepared samples.

\section{Methods \\ Mosquito samples: laboratory colonies and field-caught populations}

Non-blood-fed An. gambiae female mosquitoes were preserved in RNAlater ${ }^{\bullet} 3-5$ days post-eclosion. The following insecticide resistant strains were obtained through BEI Resources, NIAID, NIH: strain AKRON, bulk frozen, MRA-913B, contributed by Martin Akogbeto; strain RSP, bulk frozen, MRA-334 and the strain ZANU MRA-594, both contributed by Hilary Ranson and Frank H. Collins. The VK7 and Tiassalé strains were kindly provided by the Liverpool Insect Testing Establishment (LITE). The AKRON (MRA-913) strain carries the L1014F $k d r$ and G119S Ace-1 target site mutations which leads to phenotypic resistance to carbamate [23]. The RSP (MRA-334) strain's name stands for reduced susceptibility to permethrin, which is caused by the L1014S $k d r$ mutation and increased cytochrome P450 and beta-esterase activity $[24,25]$. The metabolic resistance to DDT of the ZANU (MRA-594) strain is conferred by elevated glutathionine-S-transferase and beta-esterase activity [26]. The An. gambiae strain from Tiassalé in Côte d'Ivoire exhibits the L1014F $k d r$ and G119S Ace-1 mutations as well as upregulation of several P450s [3, 27]. The combination of these different resistance mechanisms leads to multiple-insecticide resistance to pyrethroids (documented for permethrin and deltamethrin), organochlorides (DDT), carbamates (bendiocarb) and organophosphates (fenitrothion) in the Tiassalé strain. The VK7 strain's high resistance to pyrethroids and DDT is a consequence of mutations in the common target site of these insecticides. The effect of the fixed L1014F $k d r$ mutation is enhanced by the N1575Y super- $k d r$ mutation in a substantial proportion of the VK7 colony [28]. The Kisumu and Ngusso laboratory colonies are susceptible to all above mentioned insecticides and were used as control comparator strains in this study. The insectary at the Swiss Tropical and Public Health Institute provided specimens of the Kisumu colony that originates from an MRA-762 egg batch provided by BEI Resources [29]. The Ngusso specimens used in this study were reared in the insectary of the Institute of Molecular Biology and Biotechnology, Foundation for Research and Technology-Hellas. The description of the characteristics of the laboratory colonies included in the study is summarised in Additional file 1: Table S1. Specimens from a field-caught population from Bioko Island that 
was recently characterised [10] were also included in the validation of the assays.

\section{Nucleic acid extractions and preparation of samples omitting the extraction step}

Mosquitoes (10 individuals per sample) were mechanically disrupted using a tissue grinder and pestle in a 1.5 $\mathrm{ml}$ microcentrifuge tube with $200 \mu \mathrm{l}$ TE buffer $(10 \mathrm{mM}$ Tris- $\mathrm{HCl}, 1 \mathrm{mM}$ EDTA, $\mathrm{pH}$ 8.0). Nucleic acids (total RNA and DNA) were extracted using the MagnaMedics magnetic-bead based protocol (MagnaMedics $\mathrm{GmbH}$, Aachen, Germany). In brief, $150 \mu$ l lysis buffer were added to the previously processed mosquitoes, followed by $10 \mathrm{~min}$ incubation at room temperature and a centrifugation step at $16,000 \times g$ for $2 \mathrm{~min}$ in order to sediment non-lysed tissue debris. The clear lysate supernatant was incubated subsequently with $30 \mu \mathrm{l}$ magnetic beads and $440 \mu \mathrm{l}$ binding buffer for $10 \mathrm{~min}$ and was washed twice with $200 \mu \mathrm{l}$ wash buffer for $1 \mathrm{~min}$. Nucleic acid elution was performed with $150 \mu \mathrm{l}$ elution buffer for $10 \mathrm{~min}$ at $50{ }^{\circ} \mathrm{C}$. For the direct PCR approach, the clear lysate was directly diluted $25 \times$ with DEPC-treated water and used as a template for the RT-qPCR reactions. Smaller dilutions were also tested but the $25 \times$ dilution was selected due to both absence of inhibition and optimal sensitivity. Nucleic acid integrity was assessed via agarose gel electrophoresis $(1.2 \% \mathrm{w} / \mathrm{v})$.

\section{Reverse transcription and singleplex qPCR based on SYBR Green chemistry}

As a reference method to measure gene expression, singleplex qPCR assays based on SYBR Green chemistry were performed. cDNA was synthesized using $1 \mu \mathrm{g}$ of total RNA, previously treated with $\mathrm{TURBO}^{\mathrm{Ts}}$ DNase (Invitrogen, Carlsbad, CA, USA), with oligo (dT)12-18 primers and the Thermoscript RT-PCR system kit (Invitrogen, Carlsbad, CA, USA), following the manufacturer's instructions. The SYBR Green-based qPCR assays were run in duplicates in $10 \mu \mathrm{l}$ reactions, consisting of $2 \times$ Kapa SYBR $^{\oplus}$ Fast Universal qPCR Master Mix (Kapa Biosystems, Wilmington, MA, USA), forward and reverse primers specific for each gene (Additional file 1: Table S2) at a final concentration of $200 \mathrm{nM}$ as well as 20 ng of cDNA template. Bio-Rad CFX Connect ${ }^{\text {тM }}$ Real-Time PCR Detection was used with a thermal protocol consisting of a $3 \mathrm{~min}$ polymerase activation/initial denaturation step at $95{ }^{\circ} \mathrm{C}, 40$ cycles of denaturation and annealing/extension steps at $95{ }^{\circ} \mathrm{C}$ for $3 \mathrm{~s}, 60{ }^{\circ} \mathrm{C}$ for $30 \mathrm{~s}$, followed by a melting curve analysis step. A no-template control was included in each qPCR run.

\section{Design of multiplex detox assays}

A total of four triplex detox assays [Detox (A)-Detox (D)] were designed using the three fluorophores, FAM (green),
HEX (yellow) and Atto647N (red) of the TaqMan probe chemistry. Along with the 40S ribosomal protein S7 (RPS7; AGAP010592), CYP6P3 (AGAP002865) and CYP6M2 (AGAP008212) compiled Detox (A), CYP9K1 (AGAP000818) and CYP6P4 (AGAP002867) Detox (B), CYP6Z1 (AGAP008219) and GSTE2 (AGAP009194) Detox (C) and CYP6P1 (AGAP002868) and CYP4G16 (AGAP001076) Detox (D). The RPS7 target has previously shown to be a suitable reference in An. gambiae [5, 6] and was included in all assays to normalise each reaction for variations in RNA concentrations.

Primers and probes for the multiplex TaqMan qPCR assays were designed de novo (Additional file 1: Table S2). In addition to the standard guidelines for qPCR assays the following criteria were considered: (i) at least one primer was chosen to bridge the junction of two exons to avoid DNA amplification (Additional file 2: Figure S1); (ii) specificity for each gene obtained both by amplification (primers) and detection (probe) properties; and (iii) avoiding cross-reactions with other targets in the same multiplex.

Inclusivity was assessed in silico to ensure that the newly developed triplex assays detect all sequences of the target genes in An. gambiae. All available sequences for each of the nine target genes were downloaded from the NCBI Nucleotide database and analysed for matches with the corresponding assay using the "Test with saved primers" tool in Geneious 10.2.5 (Biomatters Ltd, Auckland, New Zealand) [30].

In order to assess the exclusivity in terms of sequences and the sibling species of the An. gambiae complex, the primers and probes included in the assays from each multiplex were analysed in silico. The Perl script Simulate_PCR [31] was used together with BLAST+ 2.7.1 [32]. Briefly, a list with all primers and probes was used to perform a BLAST search against two different databases: the nt database (downloaded from the NCBI BLAST ftp site, version from 08/06/2018) and a list with all DNA sequences available for mosquitoes of the An. gambiae species complex in VectorBase [33]. The Simulate_PCR script analyses the BLAST output to look for pairs of primers that could produce an amplicon and only oligos with 4 or fewer mismatches with the target sequence were considered. The amplicons were also only considered if the size was between $40 \mathrm{bp}$ and $500 \mathrm{bp}$. With the software it was also checked that a probe bound within the potential amplicons.

Primers were also analysed with the software Autodimer-1 [34] for the potential formation of primer dimers between those pairs present in the same multiplex assay. The software calculates a score based on the number of matches $(+1)$ and mismatches $(-1)$ between 2 oligos. The recommended threshold of 7 for this score was used without any potential dimer found. Further 
tests were performed with more liberal conditions and the most stable of the interactions identified had a $\mathrm{T}_{\mathrm{m}}$ of $29.7{ }^{\circ} \mathrm{C}$ and a deltaG of $-3.96 \mathrm{kcal} / \mathrm{mole}$. These interactions were deemed not relevant as the lowest temperature used during the qPCR is $60{ }^{\circ} \mathrm{C}$ and the regular threshold for deltaG on primer dimers is $-5 \mathrm{kcal} / \mathrm{mole}$.

\section{Multiplex RT-qPCR Taqman assays}

All oligos were optimised in terms of reaction efficiency and sensitivity by prioritising on those oligos that yielded an early $\mathrm{Ct}$ value at the lowest concentration. To facilitate the selection process, the reactions with combinations of oligos at different concentrations were carried out and plotted in a heat diagram (Additional file 2: Figure S2) and the pairs that gave maximum yield with the lowest concentrations were chosen. After primer optimisation a range of probe concentrations were also tested following the same criterion as well as fluorescence signal strength. To verify the absence of crosstalk and background signal, each target was amplified individually using corresponding plasmid controls. Additionally, all assays were run comparatively on the same plate in triplex and singleplex formats. The one-step reverse transcription qPCR (RT-qPCR) mastermix that was used in this study was supplied by Fast Track Diagnostics (Esch-sur-Alzette, Luxembourg). Nucleic acids of at least $100 \mathrm{ng}$ per sample were used in a total reaction volume of $10 \mu \mathrm{l}$ with the primer-probe concentrations given in Additional file 1: Table S2. The thermal cycle parameters were: $50{ }^{\circ} \mathrm{C}$ for $15 \mathrm{~min}, 95{ }^{\circ} \mathrm{C}$ for $3 \mathrm{~min}$, and 40 cycles of $95{ }^{\circ} \mathrm{C}$ for $3 \mathrm{~s}$ and $60{ }^{\circ} \mathrm{C}$ for $30 \mathrm{~s}$. The reactions were performed in 96-well plates in a ViiA 7 Real-Time PCR System (Applied Biosystems, Waltham, MA, USA). Samples were amplified in duplicates and each run always included a non-template control.

The sensitivity and specificity of the RT-qPCR assays developed were evaluated through quality control procedures consisting of: (i) the construction of standard curves for all genes both in singleplex and in multiplex formats; (ii) including a control sample consisting of RNase treated template to ensure only mRNA and no gDNA is amplified; (iii) visualisation of the amplicons by agarose gel electrophoresis for all amplicons to check for the presence of a unique band with the expected length of each amplicon's mRNA (Additional file 2: Figure S3); and (iv) estimating assay reproducibility, expressed by the coefficient of variation $(\mathrm{CV})$, by analysing a series of samples within the same and different runs. The analytical parameters of the RT-qPCR reactions are presented in details in Table 1.

Following quality control, the newly-developed multiplex detox assays were used to measure gene expression in laboratory colonies for which data were available, from the literature and/or from publicly available databases for the expression profiles of the genes included in our assays. Akron, a multi-resistant laboratory colony [35], was also used in this analysis. The same susceptible laboratory strain as reported in each published study/database was used for comparison. Additionally, the standard two-step singleplex RT-qPCR with SYBR Green chemistry was performed for each gene and the \% difference between the fold changes measured by the multiplex and singleplex approaches was calculated as described in the Bland Altman analysis [36].

\section{Statistical analysis}

Calculation of fold changes and $P$-values were assessed by the method of Pfaffl et al. [37], implemented in the REST 2009 software v2.013 that allows inputting Ct values for controls (susceptible population) and samples (resistant population) for each gene and returning fold changes for the resistant population with $95 \%$ confidence intervals (CIs) and $P$-values. The $\mathrm{Ct}$ values of normaliser and each target gene for each sample are jointly reallocated to susceptible and resistant groups and the fold changes are calculated on the basis of mean values after 2000 iterations. This statistical model described by Pfaffl et al. (Pair Wise Fixed Reallocation Randomization test $\odot$ ) [37] has the advantage of making no distributional assumptions and at the same time do not suffer a reduction in power relative to standard parametric tests. Graphs were produced using SigmaPlot v12.0 software. For the correlation analysis between expression levels, the latter were calculated as relative quantification $(\mathrm{RQ})$ units $\left(\mathrm{RQ}=2^{-\Delta \mathrm{Ct}}\right.$, where $\left.\Delta \mathrm{Ct}=\mathrm{Ct}_{\text {target }}-\mathrm{Ct}_{\text {normaliser }}\right)$. The level of significance was set at $\alpha=0.05$.

\section{Results}

Control of quality, analytical performance and of multiplex detox assays. Demonstration of expression analysis

The in silico inclusivity analysis predicted that all mRNA sequences available for all genes and their isoforms, where applicable, were bound by the corresponding oligonucleotides with either perfect matching or no more than one mismatch in either the reverse or the forward primer. This suggests that all sequences will be detectable with these new assays. Exclusivity analysis showed that only the nine expected targets and no other sequence are amplifiable and detectable with the new triplex assays in $A n$. gambiae (s.l.). No amplicon with oligonucleotides from different assays is expected and the sequence analysis found no potential primer dimers between the oligos present in the same multiplex mix.

Results from the standard curve analysis showed similar and within the accepted range [38] efficiencies (95-109\%) and $R^{2}$ values (0.989-0.999) both for single- 
Table 1 Quality control characteristics of the qPCR reactions

\begin{tabular}{|c|c|c|c|c|c|c|c|c|}
\hline Gene (assay) & \% Reaction efficiency (M) & \% Reaction efficiency (S) & $R^{2}(M)$ & $R^{2}(S)$ & Dynamic range $(\mathrm{M})$ & Dynamic range $(\mathrm{S})$ & $\% \mathrm{CV}(\mathrm{M})$ & $\% C V(S)$ \\
\hline RPS7 (Detox A-D) & 104 & 103 & 0.999 & 0.998 & $16.50-31.00$ & $16.20-31.00$ & 8.89 & 9.52 \\
\hline CYP6P3 (Detox A) & 107 & 102 & 0.999 & 0.991 & $25.70-32.92$ & $25.96-33.46$ & 9.99 & 12.37 \\
\hline CYP6M2 (Detox A) & 109 & 103 & 0.998 & 0.996 & 26.39-32.97 & $26.44-34.31$ & 9.64 & 10.41 \\
\hline CYP9K1 (Detox B) & 105 & 101 & 0.994 & 0.989 & $20.22-31.67$ & $20.74-32.02$ & 6.50 & 9.64 \\
\hline CYP6P4 (Detox B) & 109 & 107 & 0.996 & 0.996 & $25.54-33.94$ & $25.83-34.30$ & 4.46 & 10.41 \\
\hline CYP6Z1 (Detox C) & 99 & 95 & 0.995 & 0.997 & $23.37-33.63$ & $23.29-33.29$ & 5.54 & 9.99 \\
\hline GSTE2 (Detox C) & 100 & 96.1 & 0.999 & 0.998 & $23.25-33.36$ & $23.62-33.69$ & 12.07 & 16.03 \\
\hline CYP6P1 (Detox D) & 97 & 95 & 0.997 & 0.996 & $24.66-34.17$ & $24.87-34.27$ & 10.37 & 13.55 \\
\hline CYP4G16 (Detox D) & 100 & 98 & 0.999 & 0.998 & $19.22-30.30$ & $19.52-30.40$ & 9.02 & 12.24 \\
\hline
\end{tabular}

Abbreviations: $M$, multiplex; $S$, singleplex; $C V$, coefficient of variation at the expression units level

and multi-plex reactions for each gene. Assay reproducibility ranged from $\% \mathrm{CV}=4.46-12.07 \%$ for multiplex and $\% \mathrm{CV}=9.52-16.03 \%$ for singleplex reactions. The quality control data are described in detail in Table 1 . No detectable signal was obtained from DNA samples, verifying the validity of primer design across junctions (Additional file 2: Figure S1). The presence of a unique band with the expected length of each amplicon's mRNA was verified and additional specificity was achieved with probe hybridisation specific for each gene (Additional file 2: Figure S3 and Additional file 2: Figure S1).

As an example of the expression analysis output obtained with the new multiplex TaqMan assays, Fig. 1 shows that the multiple-insecticide resistant Akron strain overexpresses all eight detoxification genes compared to the susceptible Kisumu strain. In Fig. 1a-d, the amplification curves of all genes belonging to the four triplex assays are shown; it is clear that for all detoxification genes there are lower $\mathrm{Ct}$ values in the resistant compared to the susceptible sample, whereas no change was observed for the RPS7 reference gene, indicating a clear upregulation of the target (detoxification) genes. In Fig. 1e-h the fold-upregulation for gene is presented along with $95 \% \mathrm{CIs}$ and $P$-values produced as a simple output by the REST 2009 software.

\section{Validation of the diagnostic performance of multiplex detox assays using laboratory mosquito colonies}

Overall, the newly developed multiplex TaqMan assays produced comparable results with the standard two-step singleplex RT-qPCR method (Table 2). The expression levels defined by the two methods showed a statistically significant correlation for all genes. The overall \% difference in fold change determination between the two methods was $-17.6 \%$, and no significant bias was detected $(95 \%$ CI of $\%$ difference $=-38.7-3.43 \%)$.

For each individual gene, a direct comparison of the results obtained from the novel multiplex TaqMan assays, standard singleplex SYBR Green based qPCR and publicly available data is presented in Table 2. CYP6P3 and CYP6M2 were upregulated in the Tiassalé $v s$ Ngousso comparison in the literature (29.5x and 6.41 $\times$ ), the singleplex RT-qPCR (15.1× and 4.99x) and the multiplex Detox (A) panel (20.7x and 4.46x). Both genes were also upregulated in VK7 compared to Ngousso. CYP9K1 and CYP6P4 were upregulated 4.33x and $14.4 \times$ in Tiassale $v s$ Ngousso according to the literature, which is consistent with the $2.01 \times$ and $13.0 \times$ upregulation observed using the Detox (B) panel, and which was further confirmed with singleplex qPCR (4.60x and $8.33 \times$ upregulation). Similarly, CYP6Z1 and GSTE2 have been previously identified to be significantly overexpressed (3.50- and 7.80-fold) in both RSP and ZANU resistant strains when compared with the susceptible Kisumu strain and this observation is validated both by the newly developed multiplex Detox assay (C) panel (3.03- and 57.8 -fold) and the singleplex qPCR (2.69- and 22.7-fold). According to published data CYP6P1 and CYP4G16 are marginally upregulated in the resistant VK7 when compared to the susceptible Ngousso strain (2.20- and 1.90 -fold, respectively). This marginal difference in gene expression is also observed when assaying the same strains with the Detox assay (D) panel (1.55- and 2.03-fold) and singleplex qPCR (2.64- and 2.63-fold).

Additionally, differences that are not statistically significant according to published data and the standard singleplex RT-qPCR are also concordant with the multiplex detox assays. These negative predictive properties of our method were found for CYP6Z1, GSTE2 and CYP4G16 in Tiassalé vs Ngousso comparisons and CYP9K1 in VK7 vs Ngousso comparison.

\section{Validation of the diagnostic performance of multiplex detox assays with field-caught specimens}

In addition to the experiments above, RNA templates from two deltamethrin-resistant field populations, hereafter named "Industrial" and "Hospital", from the Ela Nguema district of Malabo in Bioko (described in detail 


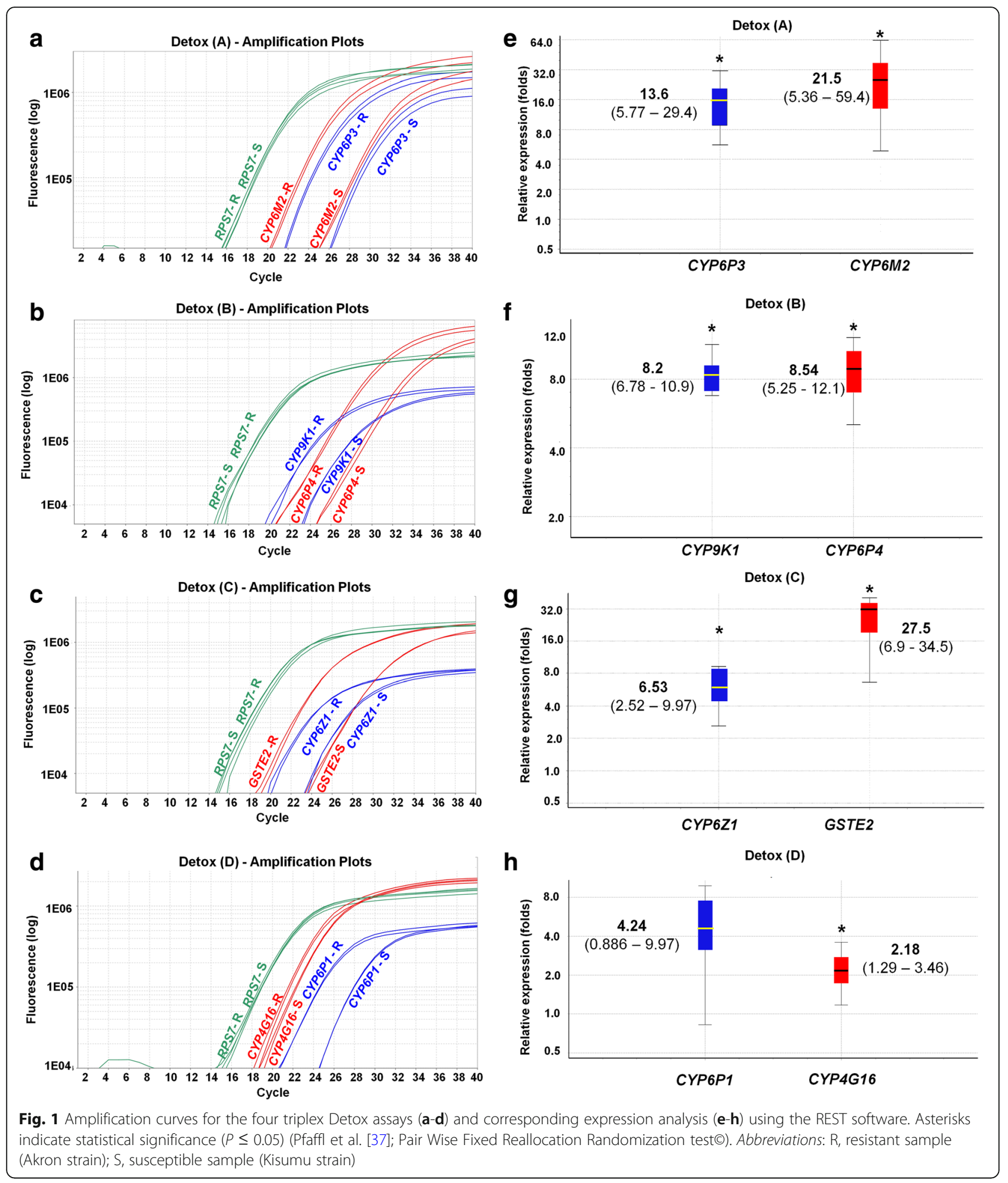

in [10]) were included in this study. Here, the idea was to further validate the multiplex approach against additional methods, two step singleplex RT-qPCR and DNA microarrays, by measuring the fold changes in expression levels of the eight detoxification genes between the field mosquitoes and a susceptible Kisumu lab colony [10]. Indeed, the estimated fold changes between the two Bioko field populations and the susceptible lab colony were in good agreement between all three methods (Fig. 2). For example, CYP9K1 showed a 4.21-fold 


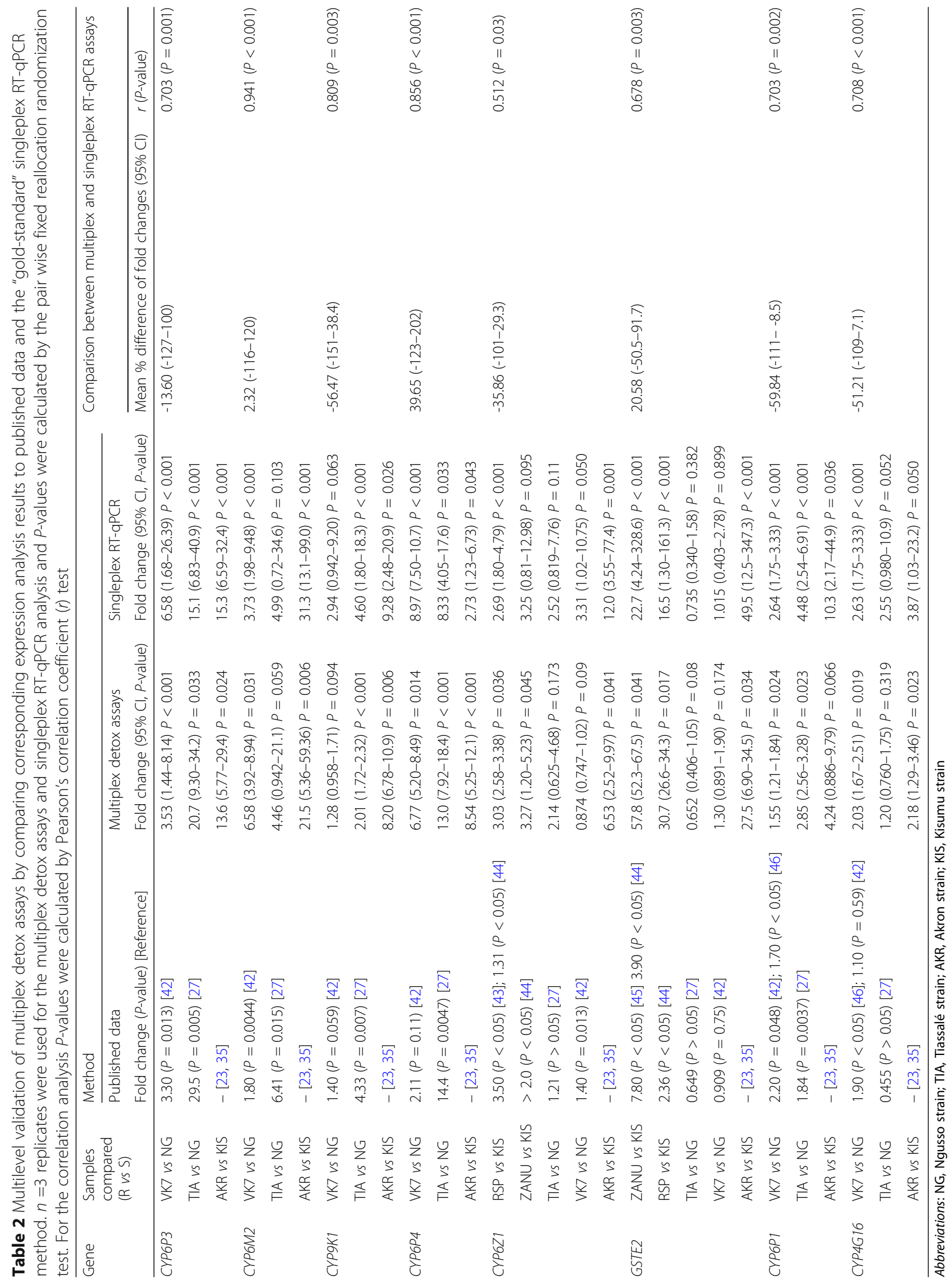



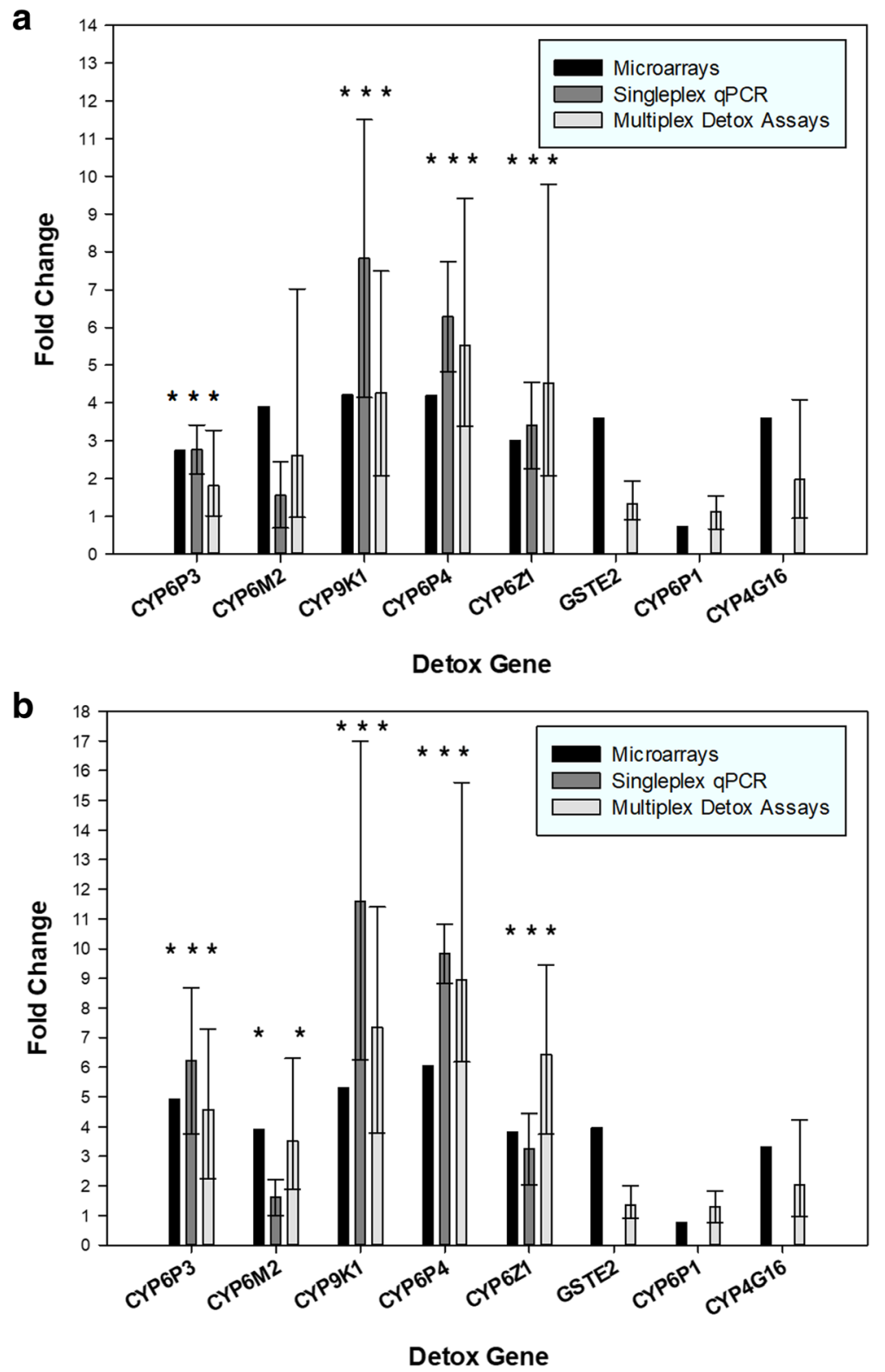

Fig. 2 Expression analysis in field-caught samples from Bioko Industrial (a) and Hospital (b) areas vs the Kisumu susceptible strain $(n=3$ replicates each), showing accordance between the multiplex detox assays and two different methodologies (microarrays and SYBR Green singleplex RTqPCR). Levels in error bars indicate $95 \% \mathrm{Cls}$; asterisks indicate statistical significance $(P \leq 0.05)$

upregulation in the "Industrial" population as shown by the microarrays of the initial study (Fig. 2a). In the two step singleplex RT-qPCR assays the fold change was 7.82 (95\% CI: 4.14-11.5) and the current assays yielded a 4.27-fold (95\% CI: 2.07-7.49) change. In the Hospital population the microarray output reports a 5.3-fold overexpression for CYP9K1 (Fig. 2b), which agrees with the 7.35-fold (95\% CI: 3.79-11.4) upregulation found with the multiplex detox assays and 11.6-fold (95\% CI: 6.25-17.0) in the two step singleplex RT-qPCR method.
Similarly, highly consistent comparative results between the three methods were obtained for CYP6P3, CYP9K1, CYP6P4, CYP6M2 and CYP6Z1 for both the Industrial (Fig. 2a) and Hospital (Fig. 2b) field populations. The complete expression analysis results are presented in Additional file 1: Table S3 for the Industrial area and in Additional file 1: Table S4 for the Hospital area population.

For GSTE2, CYP6P1 and CYP4G16 no singleplex RT-qPCR data are available because they were not followed up in the original study given the microarrays 
showed no differential expression. These observations are confirmed by our method.

Ultra-rapid and cost-efficient application of the validated multiplex detox assays with the direct RT-qPCR approach In order to provide an even more rapid and cost-effective format for analysing detoxification gene expression levels, the possibility of omitting the nucleic acid extraction step was assessed. In order to measure the effect of omitting the extraction step, mosquitoes from the susceptible Kisumu and the resistant Akron colonies were homogenised and lysed before they were divided into two aliquots. Nucleic acids were extracted from the one aliquot, while the other aliquot served directly as a RT-qPCR template after it was further diluted at a 1:25 ratio. Expression analysis data obtained from the aliquot that was directly subjected to RT-qPCR were completely consistent with those obtained from the aliquot from which the nucleic acids were purified. For example, in the comparison between resistant and susceptible mosquitoes using the Detox (A) assay, CYP6P3 was found to be overexpressed 4.68× (95\% CI: 2.67-8.81) with the direct RT-qPCR approach compared with a 4.10-fold overexpression (95\% CI: 3.35-5.23) when following the standard nucleic acid extraction procedure. Similarly, the difference for CYP6M2 was minor between the two approaches (4.57-fold compared to 2.70-fold upregulation for direct RT-qPCR compared to standard procedure, respectively). Detailed results for all genes are presented in Table 3. To corroborate this analysis, gene expression values obtained from the two approaches were also correlated and correlation coefficients indicated a significantly strong correlation $(r>0.9$ in most cases) (Additional file 1: Table S5). Figure 3 provides an easy-to-follow three-step overview of the direct RT-qPCR procedure from sample-to-result for which only $95 \mathrm{~min}$ are needed.

\section{Discussion}

We developed a simple, robust, rapid and cost-effective method for determining the expression levels of major detoxification genes that have been associated with metabolic resistance in the malaria vector An. gambiae. The assay is based on specific TaqMan probes and RT, and it can reliably determine the expression levels of the pyrethroid metabolisers CYP6P3, CYP6M2, CYP9K1, CYP6P4, CYP6Z1 and GSTE2, which have been functionally implicated in metabolic pyrethroid resistance in An. gambiae. The assay panel is also readily expandable to capture additional loci as they evolve, or to cover other mosquito vectors.

The method was developed and successfully validated with mosquito specimens from both laboratory colonies and field collections. The results obtained are consistent (both in terms of extent of upregulation and statistical significance) with previously-used methods regarded as gold standards for gene expression determination, such as the two-step standard singleplex qPCR and microarrays, which are far more expensive and complex. The comparative analysis between multiplex TaqMan assays and SYBR Green singleplex qPCR did not reveal significant discordance for the majority of genes (Table 2) with the exception of CYP6P1 (95\% CI of \%difference: -111-8.5, not including the null value). Furthermore, compared to other genes, CYP9K1 and CYP4G16 show relatively noticeable differences in fold changes between multiplex and singleplex assays $(-56.5 \%$ and $-51.2 \%$ difference, respectively). A possible explanation for this observation could be given by the fact that CYP6P1, CYP4G16 and CYP9K1 are among the genes that did not show large or statistically significant differences in most of the available comparisons between the lab strains of Table 2, so some variability in determinations between methods is expected. However, even for these genes, available data from the third independent method (microarrays) were in every case in better agreement with the newly developed multiplex, rather

Table 3 Gene expression analysis performed in mosquito lysates by direct qPCR and matched purified eluates after nucleic acid extraction ( $n=4$ susceptible vs $n=4$ resistant). $P$ values were calculated by the pair wise fixed reallocation randomization test

\begin{tabular}{|c|c|c|c|c|}
\hline \multirow{2}{*}{$\begin{array}{l}\text { Gene } \\
\text { (Detox } \\
\text { assay) }\end{array}$} & \multicolumn{2}{|l|}{ Direct qPCR in lysates } & \multicolumn{2}{|c|}{ qPCR in matched nucleic acid extracted eluates } \\
\hline & Fold change $(95 \% \mathrm{Cl})$ & $P$-value & Fold change $(95 \% \mathrm{Cl})$ & $P$-value \\
\hline CYP6Р3 (A) & $4.68(2.67-8.81)$ & 0.001 & $4.10(3.35-5.23)$ & 0.020 \\
\hline CYPGM2 (A) & $4.57(3.18-7.19)$ & 0.002 & $2.70(2.18-3.40)$ & 0.022 \\
\hline CYP9K1 (B) & $4.66(3.40-7.70)$ & 0.002 & $4.45(4.12-4.80)$ & $<0.001$ \\
\hline CYP6P4 (B) & $2.29(1.31-3.71)$ & 0.018 & $1.67(1.33-2.11)$ & $<0.001$ \\
\hline CYP6Z1 (C) & $2.98(2.47-3.84)$ & 0.009 & $1.85(1.44-2.22)$ & $<0.001$ \\
\hline GSTE2 (C) & $13.6(9.64-18.4)$ & 0.008 & $9.36(7.96-11.9)$ & $<0.001$ \\
\hline CYP6P1 (D) & $1.32(0.968-1.90)$ & 0.060 & $1.64(0.752-3.57)$ & 0.215 \\
\hline CYP4G16 (D) & $2.81(1.61-4.26)$ & 0.008 & $1.91(1.45-2.46)$ & $<0.001$ \\
\hline
\end{tabular}




\section{Process overview: from mosquito samples to differential expression results}

\section{STEP 1}

(a) Add lysis buffer and homogenise mosquitoes stored in RNAlater

(b) Incubate for $10 \mathrm{~min}$

(c) Collect clear supernatant after

brief high- speed centrifugation.

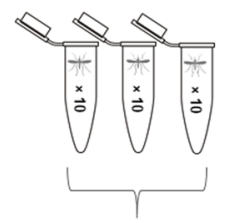

Susceptible (S) mosquitoes $3 \times$ replicates

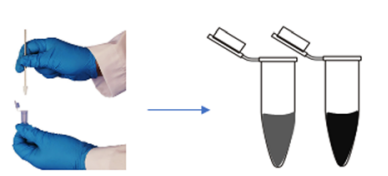

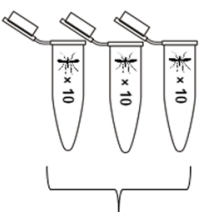

Resistant (R) mosquitoes $3 \times$ replicates

\section{STEP 2}

Use diluted (1:25) lysate for multiplex RTqPCR

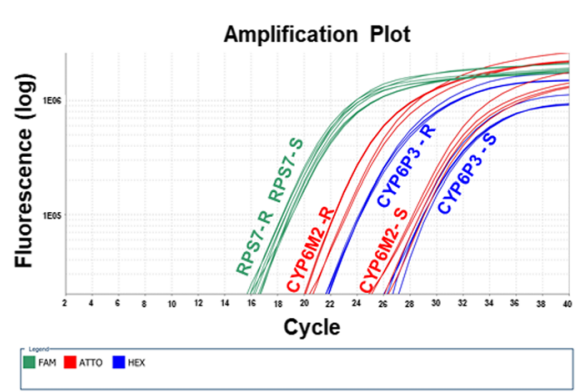

Step Process

1 Homogenisation-Lysis-Centrifuge-Dilute Lysate

2 Prepare and run RT-qPCR

3 Perform statistical analysis

(1) Total time to result $=95 \mathrm{~min}$

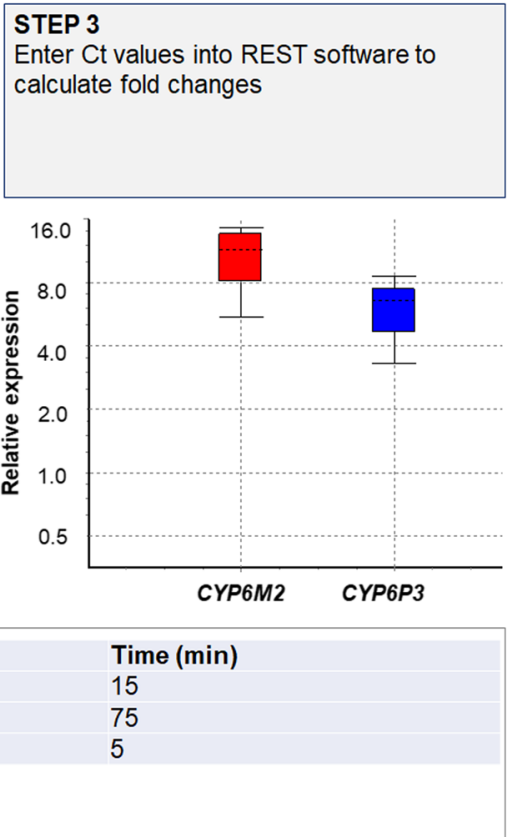

Fig. 3 A three-step process from sample lysis to gene expression analysis for two populations (Resistant vs Susceptible, $n=3$ replicates each). For the abovementioned samples a total-time-to-results of approximately 95 min is needed

than the singleplex assays (Table 2, Additional file 1: Table S3 and Additional file 1: Table S4). All points above suggest that our multiplex assays represent a valid alternative to conventional two-step singleplex qPCR. The "closed-tube" nature of the TaqMan platform means that there is no requirement for post-PCR processing and consequently assays are simple to perform and rapid to run, while the output is easy to produce and interpret. A necessary requirement for these triplex-assay is a real-time PCR machine equipped with three detection channels. Such machines are generally more expensive (approximate price range $=20,000-25,000 €$ ) than machines with only two detection channels (approximate price range = $10,000-15,000 €)$, but there is currently fast progress in the development of cheap portable field-deployable real-time qPCR thermocyclers. The investment in a flexible multi-channel qPCR machine is worthwhile because multiplex qPCR assays have much lower running cost. In Table 4 we estimated the cost for measuring the gene expression levels of the eight detox genes and one normaliser gene for 12 samples. For the singleplex approach at least $2.25 \times$ more mastermix and plastic consumables are needed than for the multiplex assay, while the multiplex method only requires additional TaqMan probes that cost roughly $10 €$ extra per $96-$-well plate. The total consumables and reagents for the multiplex TaqMan assay cost approximately half as much as for the same tests with the singleplex SYBR Green assay.
Furthermore, the presented assays run with mosquito lysates without the requirement of prior RNA extraction and DNase treatment steps due to the primer design across exons. The results suggest that there is little, if any, loss in sensitivity, compared to standard nucleic acid extraction approaches. The extraction-free multiplex RT-qPCR method is easier to perform than the singleplex assays, reliable and rapid, with a total time from sample-to-result as short as $95 \mathrm{~min}$. The addition of the extraction step alone would add an additional $1 \mathrm{~h}$ approximately and the performance of assays with the singleplex format would significantly increase the time to result even further (preparation of three reactions instead of one

Table 4 Cost comparison of triplex TaqMan vs singleplex SYBR Green approach. This is a rough cost estimation for measuring the gene expression levels of eight detox genes and one normaliser gene in 12 samples on a standard 96-well plate format

\begin{tabular}{lll}
\hline $\begin{array}{l}\text { Consumables and } \\
\text { reagents }\end{array}$ & $\begin{array}{l}\text { TaqMan triplex } \\
\text { RT-qPCR }\end{array}$ & $\begin{array}{l}\text { SYBR Green } \\
\text { singleplex RT-qPCR }\end{array}$ \\
\hline $\begin{array}{l}\text { 96-well plastic } \\
\text { plate and optical film }\end{array}$ & $1 \times 6.50 €=6.50 €$ & $3 \times 6.50 €=19.50 €$ \\
Mastermix & $1 \times 25.00 €=25.00 €$ & $2.25 \times 25.00 €=56.25 €$ \\
Primers & $1 \times 0.50 €=0.50 €$ & $2.25 \times 0.50 €=1.13 €$ \\
TaqMan Probes & $1 \times 10.00 €=10.00 €$ & - \\
Total cost & $42.00 €$ & $76.88 €$ \\
\hline
\end{tabular}


and possibly more than one qPCR run required dependent on the number of samples). The direct-in-lysates RT-qPCR approach seems to produce more variable results (indicated by broader 95\% CIs) compared to the standard RNA extraction method and thus more technical replicates might be required to achieve similar precision.

Our method has important features for the future development of diagnostic kits. It does not require a cold chain, as it can be used with samples preserved in RNAlater ${ }^{\circ}$ and could be performed with ready-to-use lyophilised RT-PCR enzyme/primer/probe pellets. It is also flexible to include additional genetic traits for insecticide resistance as they evolve and to expand to other species. Furthermore, the format we suggest could include other diagnostic markers such as species identification [39] and Plasmodium detection [40] markers. Thus, the multiplex assays described here along with the suggested expansions represent ideal candidates for incorporation in automated diagnostic platforms for mosquito vector surveillance such as the DMC-MALVEC-LabDisk currently under development [22].

\section{Conclusions}

The novel multiplex qRT-PCR assays for monitoring the expression levels of genes previously associated with metabolic resistance in An. gambiae (s.l.) are simple to perform, robust, rapid, cost-effective and work with RNAlater preserved field-collected mosquitoes that can be shipped at room temperature. The approach we suggest here is, therefore, relevant and suitable for insecticide resistance management (IRM) programmes, on the one hand because large numbers of specimens can be handled without extra care, and on the other hand because the speed of the assay allows for producing data as required within short time. The specificity of P450-based metabolic resistance against some but not other active ingredients, as well as the "unexpected case" of cross-resistance against different groups of insecticides [41], indicates that the development of diagnostic tools able to detect specific detoxification enzyme-based resistance is important. Together with bioassays and molecular tests for target site resistance detection, our assays could identify resistance at an early stage and guide efficient and sustainable IRM strategies.

\section{Additional Files}

Additional file 1: Table S1. List of laboratory strains and their characteristics. Table S2. Primers and probes used in the study. Table S3. Expression analysis in field-caught samples from Bioko (Industrial area) vs Kisumu susceptible strain. Table S4. Expression analysis in field-caught samples from Bioko (Hospital area) vs Kisumu susceptible strain. Table S5. Accordance between expression levels measured in mosquito lysates by direct $\mathrm{GPCR}$ and purified eluates after nucleic acid extraction $(n=8)$ (DOCX 29 kb)

Additional file 2: Figure S1. Primer and probe design strategy using Detox (A), consisting of RPS7 (normaliser), CYP6P3 (target gene 1), CYP6M2 (target gene 2) as an example. For each gene either the forward (RPS7 and CYP6P3) or the reverse primer (CYP6M2) spanned two exons in order to avoid DNA amplification. Boxes indicate exons, lines indicate introns. Abbreviations: $F$, forward primer; $R$, reverse primer; $P$, TaqMan probe labelled with different dyes for each gene; bp, base pairs. Figure S2. Results from the primer matrices experiments for each individual gene. Ct values are plotted versus forward and reverse primer concentration concentrations. " $X$ " indicates the selected combination of forward and reverse primer concentrations by using as criterion the lowest concentration that gives the earliest Ct values. Figure S3. Agarose gel $(2.0 \% \mathrm{w} / \mathrm{v})$ electrophoresis indicating the specificity of the study's assays. Additional specificity is achieved with probe hybridisation (TaqMan chemistry) (DOCX 3803 kb)

\section{Abbreviations}

$\mathrm{Cl}$ : Confidence interval; CV: Coefficient of variation;

DDT: Dichlordiphenyltrichlorethan; IR: Insecticide resistance; IRM: Insecticide resistance management; RT-qPCR: Reverse transcription qPCR

\section{Acknowledgements}

The following reagent was obtained through BEI Resources: ZANU (MRA594), RSP (MRA-334), Akron (MRA-913). We specially thank Dr Paul Howell from CDC for organising and sending the abovementioned samples. We thank Fast Track Diagnostics (FTD) for supplying us with the RT-PCR mastermix and Liverpool Insect Testing Establishment (LITE) for providing the VK7 and Tiassalé Anopheles gambiae strains.

\section{Funding}

This project was funded by the European Union Horizon 2020 Framework Programme (688207-DMC-MALVEC).

\section{Availability of data and materials}

Data supporting the conclusions of this article are included within the article and its additional files. The datasets used and/or analysed during the present study are available from the corresponding author upon reasonable request.

\section{Authors' contributions}

JV and KM conceptualised and designed the study. KM performed the experiments and the statistical analysis. KM and JV drafted manuscript with input from all authors. NW provided and prepared samples for the study. NW and PM critically reviewed and amended the manuscript. SM and IE performed the quality control of primer/probe multiplexing. All authors read and approved the final manuscript.

Ethics approval and consent to participate Not applicable.

\section{Consent for publication}

Not applicable.

\section{Competing interests}

The authors declare that they have no competing interests.

\section{Publisher's Note}

Springer Nature remains neutral with regard to jurisdictional claims in published maps and institutional affiliations.

\section{Author details}

${ }^{1}$ Institute of Molecular Biology and Biotechnology, Foundation for Research and Technology-Hellas, 70013 Heraklion, Greece. ${ }^{2}$ Department of Epidemiology and Public Health, Swiss Tropical and Public Health Institute, Socinstrasse 57, P.O. Box, CH-4002 Basel, Switzerland. ${ }^{3}$ University of Basel, Petersplatz 1, P.O. Box, CH-4001 Basel, Switzerland. ${ }^{4}$ Fast Track Diagnostics, a Siemens Healthineers Company, Esch-sur-Alzette 4354, Luxembourg. 
${ }^{5}$ Pesticide Science Laboratory, Department of Crop Science, Agricultural University of Athens, 11855 Athens, Greece.

\section{Received: 24 August 2018 Accepted: 3 December 2018 Published online: 06 January 2019}

\section{References}

1. Bhatt S, Weiss DJ, Cameron E, Bisanzio D, Mappin B, Dalrymple U, et al. The effect of malaria control on Plasmodium falciparum in Africa between 2000 and 2015. Nature. 2015;526:207-11.

2. Ranson H, N'Guessan R, Lines J, Moiroux N, Nkuni Z, Corbel V. Pyrethroid resistance in African anopheline mosquitoes: what are the implications for malaria control? Trends Parasitol. 2011;27:91-8.

3. Edi CV, Koudou BG, Jones CM, Weetman D, Ranson H. Multiple-insecticide resistance in Anopheles gambiae mosquitoes, southern Côte d'Ivoire. Emerg Infect Dis. 2012;18:1508-11.

4. Liu N. Insecticide resistance in mosquitoes: impact, mechanisms, and research directions. Annu Rev Entomol. 2015;60:537-59.

5. Muller P, Warr E, Stevenson BJ, Pignatelli PM, Morgan JC, Steven A, et al. Field-caught permethrin-resistant Anopheles gambiae overexpress CYP6P3, a P450 that metabolises pyrethroids. PLOS Genet. 2008;4:e1000286.

6. Stevenson BJ, Bibby J, Pignatelli P, Muangnoicharoen S, O'Neill PM, Lian LY, et al. Cytochrome P450 6M2 from the malaria vector Anopheles gambiae metabolizes pyrethroids: sequential metabolism of deltamethrin revealed. Insect Biochem Mol Biol. 2011;41:492-502.

7. Chiu TL, Wen Z, Rupasinghe SG, Schuler MA. Comparative molecular modeling of Anopheles gambiae CYP6Z1, a mosquito P450 capable of metabolizing DDT. Proc Natl Acad Sci USA. 2008;105:8855-60.

8. Ibrahim SS, Riveron JM, Stott R, Irving H, Wondji CS. The cytochrome P450 CYP6P4 is responsible for the high pyrethroid resistance in knockdown resistance-free Anopheles arabiensis. Insect Biochem Mol Biol. 2016:68:23-32.

9. Yunta C, Grisales N, Nasz S, Hemmings K, Pignatelli P, Voice M, et al. Pyriproxyfen is metabolized by $\mathrm{P} 450$ s associated with pyrethroid resistance in An. gambiae. Insect Biochem Mol Biol. 2016;78:50-7.

10. Vontas J, Grigoraki L, Morgan J, Tsakireli D, Fuseini G, Segura L, et al. Rapid selection of a pyrethroid metabolic enzyme CYP9K1 by operational malaria control activities. Proc Natl Acad Sci USA. 2018;115:4619-24.

11. Ranson H, Jensen B, Wang X, Prapanthadara L, Hemingway J, Collins FH. Genetic mapping of two loci affecting DDT resistance in the malaria vector Anopheles gambiae. Insect Mol Biol. 2000;9:499-507.

12. Riveron JM, Yunta C, Ibrahim SS, Djouaka R, Irving H, Menze BD, et al. A single mutation in the GSTe2 gene allows tracking of metabolically based insecticide resistance in a major malaria vector. Genome Biol. 2014;15:R27.

13. Bass C, Nikou D, Vontas J, Donnelly MJ, Williamson MS, Field LM. The Vector Population Monitoring Tool (VPMT): high-throughput DNA-based diagnostics for the monitoring of mosquito vector populations. Malar Res Treat. 2010;2010:190434.

14. Donnelly MJ, Isaacs AT, Weetman D. Identification, validation, and application of molecular diagnostics for insecticide resistance in malaria vectors. Trends Parasitol. 2016:32:197-206.

15. Brogdon WG, MCAllister JC. Insecticide resistance and vector control. Emerg Infect Dis. 1998:4:605-13.

16. Brogdon WG. Biochemical resistance detection: an alternative to bioassay. Parasitol Today. 1989:5:56-60.

17. WHO. Test procedures for insecticide resistance monitoring in malaria vector mosquitoes. 2nd edition. Geneva: World Health Organization; 2016.

18. Vulule JM, Beach RF, Atieli FK, McAllister JC, Brogdon WG, Roberts JM, et al. Elevated oxidase and esterase levels associated with permethrin tolerance in Anopheles gambiae from Kenyan villages using permethrin-impregnated nets. Med Vet Entomol. 1999;13:239-44.

19. Brooke BD, Kloke G, Hunt RH, Koekemoer LL, Temu EA, Taylor ME, et al. Bioassay and biochemical analyses of insecticide resistance in southern African Anopheles funestus (Diptera: Culicidae). Bull Entomol Res. 2001;91: 265-72.

20. Morou E, Dowd AJ, Rajatileka S, Steven A, Hemingway J, Ranson H, et al. A simple colorimetric assay for specific detection of glutathione-S transferase activity associated with DDT resistance in mosquitoes. PLoS Negl Trop Dis. 2010;4:e808

21. Riga M, Tsakireli D, Ilias A, Morou E, Myridakis A, Stephanou EG, et al. Abamectin is metabolized by CYP392A16, a cytochrome P450 associated with high levels of acaricide resistance in Tetranychus urticae. Insect Biochem Mol Biol. 2014:46:43-53.

22. Vontas J, Mitsakakis K, Zengerle R, Yewhalaw D, Sikaala CH, Etang J, et al. Automated innovative diagnostic, data management and communication tool, for improving malaria vector control in endemic settings. Stud Health Technol Inform. 2016;224:54-60.

23. Djouaka RF, Bakare AA, Coulibaly ON, Akogbeto MC, Ranson H, Hemingway J, et al. Expression of the cytochrome P450s, CYP6P3 and CYP6M2 are significantly elevated in multiple pyrethroid resistant populations of Anopheles gambiae s.s. from southern Benin and Nigeria. BMC Genomics. 2008;9:538.

24. Lines JD, Nassor NS. DDT resistance in Anopheles gambiae declines with mosquito age. Med Vet Entomol. 1991;5:261-5.

25. Vulule JM, Beach RF, Atieli FK, Mount DL, Roberts JM, Mwangi RW. Longterm use of permethrin-impregnated nets does not increase Anopheles gambiae permethrin tolerance. Med Vet Entomol. 1996:10:71-9.

26. Prapanthadara LA, Hemingway J, Ketterman AJ. DDT-resistance in Anopheles gambiae (Diptera: Culicidae) from Zanzibar, Tanzania, based on increased DDT-dehydrochlorinase activity of glutathione S-transferases. Bull Entomol Res. 1995:85:267-74.

27. Edi CV, Djogbenou L, Jenkins AM, Regna K, Muskavitch MA, Poupardin R, et al. CYP6 P450 enzymes and ACE-1 duplication produce extreme and multiple insecticide resistance in the malaria mosquito Anopheles gambiae. PLoS Genet. 2014;10:e1004236.

28. Diabate A, Baldet T, Chandre F, Akoobeto M, Guiguemde TR, Darriet F, et al. The role of agricultural use of insecticides in resistance to pyrethroids in Anopheles gambiae s.l. in Burkina Faso. Am J Trop Med Hyg. 2002;67:617-22.

29. Reid JA. Pupal differences between species A and B of the Anopheles gambiae group from Kisumu, East Africa. Mosq Syst. 1975;7:299-302.

30. Kearse M, Moir R, Wilson A, Stones-Havas S, Cheung M, Sturrock S, et al. Geneious Basic: an integrated and extendable desktop software platform for the organization and analysis of sequence data. Bioinformatics. 2012;28:1647-9.

31. Gardner SN, Slezak T. Simulate_PCR for amplicon prediction and annotation from multiplex, degenerate primers and probes. BMC Bioinformatics. 2014;15:237.

32. Altschul SF, Gish W, Miller W, Myers EW, Lipman DJ. Basic local alignment search tool. J Mol Biol. 1990;215:403-10

33. Giraldo-Calderon Gl, Emrich SJ, MacCallum RM, Maslen G, Dialynas E, Topalis $P$, et al. VectorBase: an updated bioinformatics resource for invertebrate vectors and other organisms related with human diseases. Nucleic Acids Res. 2015:43:D707-13.

34. Vallone PM, Butler JM. AutoDimer: a screening tool for primer-dimer and hairpin structures. Biotechniques. 2004;37:226-31.

35. Mutunga JM, Anderson TD, Craft DT, Gross AD, Swale DR, Tong F, et al. Carbamate and pyrethroid resistance in the akron strain of Anopheles gambiae. Pestic Biochem Physiol. 2015;121:116-21.

36. Giavarina D. Understanding Bland Altman analysis. Biochem Med (Zagreb). 2015;25:141-51.

37. Pfaffl MW, Horgan GW, Dempfle L. Relative Expression Software Tool (REST) for group-wise comparison and statistical analysis of relative expression results in real-time PCR. Nucleic Acids Res. 2002;30:e36.

38. Bustin SA, Benes V, Garson JA, Hellemans J, Huggett J, Kubista M, et al. The MIQE guidelines: minimum information for publication of quantitative realtime PCR experiments. Clin Chem. 2009;55:611-22.

39. Bass C, Williamson MS, Wilding CS, Donnelly MJ, Field LM. Identification of the main malaria vectors in the Anopheles gambiae species complex using a TaqMan real-time PCR assay. Malar J. 2007:6:155.

40. Kefi M, Mavridis K, Simoes ML, Dimopoulos G, Siden-Kiamos I, Vontas J. New rapid one-step PCR diagnostic assay for Plasmodium falciparum infective mosquitoes. Sci Rep. 2018;8:1462.

41. Nauen R, Vontas J, Kaussmann M, Wolfel K. Pymetrozine is hydroxylated by CYP6CM1, a cytochrome P450 conferring neonicotinoid resistance in Bemisia tabaci. Pest Manag Sci. 2013;69:457-61.

42. Vector Base data, Kwiatkowska et al, Multi-insecticide resistant VK population. 2013. https://www.vectorbase.org/expression-browser/ experiment/E-MTAB-1083. Accessed 21 July 2018.

43. Nikou D, Ranson H, Hemingway J. An adult-specific CYP6 P450 gene is overexpressed in a pyrethroid-resistant strain of the malaria vector, Anopheles gambiae. Gene. 2003;318:91-102.

44. David JP, Strode C, Vontas J, Nikou D, Vaughan A, Pignatelli PM, et al. The Anopheles gambiae detoxification chip: a highly specific microarray to study metabolic-based insecticide resistance in malaria vectors. Proc Natl Acad Sci USA. 2005;102:4080-4 
45. Ding Y, Ortelli F, Rossiter LC, Hemingway J, Ranson H. The Anopheles gambiae glutathione transferase supergene family: annotation, phylogeny and expression profiles. BMC Genomics. 2003;4:35.

46. Toe KH, N'Fale S, Dabire RK, Ranson H, Jones CM. The recent escalation in strength of pyrethroid resistance in Anopheles coluzzi in West Africa is linked to increased expression of multiple gene families. BMC Genomics. 2015;16:146.

Ready to submit your research? Choose BMC and benefit from:

- fast, convenient online submission

- thorough peer review by experienced researchers in your field

- rapid publication on acceptance

- support for research data, including large and complex data types

- gold Open Access which fosters wider collaboration and increased citations

- maximum visibility for your research: over $100 \mathrm{M}$ website views per year

At BMC, research is always in progress.

Learn more biomedcentral.com/submissions 